\title{
Coupling parameter for low-temperature plasma with condensed phase
}

\author{
V.I.Vishnyakov, G.S.Dragan \\ Mechnikov Odessa National University, Odessa 65026, Ukraine
}

Received March 6, 2006, in final form May 18, 2006

\begin{abstract}
The conditions of formation of ordered structures in low-temperature plasma with the condensed disperse phase are studied. Various modifications of the coupling parameter for polydisperse systems of condensed grains are proposed.
\end{abstract}

Key words: low-temperature plasma, condensed grains, plasma crystal

PACS: 52.27.Gr, 52.27.Lw

\section{Introduction}

The detection of plasma crystals in the dusty plasma [1,2], and the ordered structures of the condensed grains in the plasma of combustion products (smoky plasma) [3,4] stimulated the research of mechanisms of the charged grains interaction in the low-temperature plasma and definition of the criteria parameters of phase transition. Thus it has been found out that the formation of the ordered structures of dust grains occurs in strongly coupled (nonideal) plasma. It has been suggested to use a Coulomb coupling parameter $\Gamma$, defined as the ratio of the potential energy of Coulomb interaction between neighboring particles and their kinetic temperature, in order to distinguish the weakly coupled plasma and the strongly coupled plasma [5-11].

The application of such a form of coupling parameter for the description of the condensed grains interactions in the smoky plasma is not always comprehensible, which is caused by a number of its specific properties. The principal distinction of the smoky plasma from discharge dusty plasma is its thermal collision nature. The displacement of ionization equilibrium in the plasma gas phase, caused by the presence of a noncompensate volume charge, is formed in plasma due to the interphase interaction [12]. The condensed grains are formed by homogeneous and heterogeneous condensation in the smoky plasma at burning. Therefore, the condensed phase contains a multimode distribution function for sizes of grains of submicron fraction. This enables us to consider the dusty and smoky plasma as two different kinds of plasma with a condensed disperse phase.

Thus, multimode distribution functions for the mentioned grain sizes and the displacement of ionization equilibrium do not make it possible to use the coupling parameter, obtained both for the dusty and smoky plasmas. The present paper is devoted to the development of a coupling parameter applicable to the description of polydisperse systems such as the smoky plasma.

\section{The state of the problem}

The Coulomb coupling parameter was suggested $[5,6]$ as the ratio of average electrostatic energy to the average kinetic energy in the spatially homogeneous one-component plasma (OCP), consisting of a single species of charged particles embedded in a uniform background of neutralizing charges,

$$
\Gamma_{\mathrm{OCP}}=\frac{(e Z)^{2}}{4 \pi \varepsilon_{0} R_{\mathrm{W}} T},
$$


where $Z$ is the charge of particles, $R_{\mathrm{W}}=(3 / 4 \pi N)^{1 / 3}$ is the Wigner-Seitz radius, $N$ is the number density of the particles.

Such a system can be realized, for example, within a highly evolved star, where the strongly degenerate electron gas creates a neutralizing background to the Coulomb interaction of ions. Then strength of the ionic interaction can be characterized by parameter equation (1). It is generally accepted to call the system nonideal, if $\Gamma \approx 1$ and strongly nonideal or strongly coupled at $\Gamma \gg 1$.

The dusty plasma contains electrons, ions and the charged dust grains. In the plasma any charge is screened by free charges of other sign. Therefore, one-component approach is incorrect. Ikezi [7] suggested considering the screening of charges in the equation for coupling parameter, using the Debye-Hückel or Yukawa potential for calculation of the potential energy of grains interaction. Then the coupling parameter for the dust grains subsystem can be presented as follows:

$$
\Gamma^{*}=\frac{\left(\mathrm{e} Z_{\mathrm{d}}\right)^{2}}{4 \pi \varepsilon_{0} R_{\mathrm{W}} T_{\mathrm{d}}} \exp (-\kappa),
$$

where $\kappa=R_{\mathrm{W}} / r_{\mathrm{D}}$ is the structural parameter, $r_{\mathrm{D}}$ is the screening length, $T_{\mathrm{d}}$ is the temperature of dust grains. Plasma crystals are formed at value $\Gamma>\Gamma_{\mathrm{c}}$ where $\Gamma_{\mathrm{c}}$ is some critical value of the coupling parameter. Numerical calculations for one-component plasma give the value $\Gamma_{\mathrm{c}} \sim 170$.

The phase diagram of crystallization in coordinates $\Gamma-\kappa$, was gained in the papers [8-11] by means of numerical modelling (figure 1). Thus it is noted that the coupling parameter in the form of equation (2) provides a better description of a phase transition.

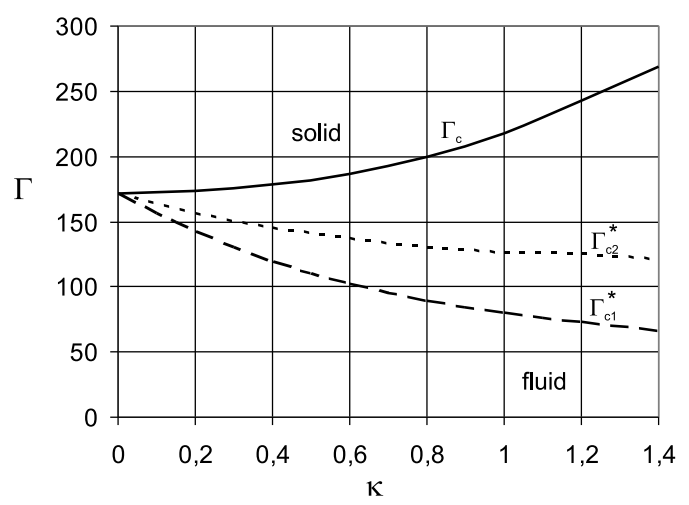

Figure 1. The phase diagram of the dust grains crystallization; $\Gamma_{c 1}^{*}=\Gamma_{\mathrm{c}} \exp (-\kappa), \Gamma_{c 2}^{*}-$ equation (3) (data from $[9,10])$.

The results of experimental research regarding the formation of plasma crystals [13] showed the necessity of modernizing the coupling parameter equation (2) to the following form

$$
\Gamma^{*}=\Gamma_{\mathrm{OCP}}(1+\kappa) \exp (-\kappa)
$$

The further researches [14-17] showed the necessity of improving the coupling parameter [17],

$$
\Gamma^{*}=\Gamma_{\mathrm{OCP}}\left(1+\kappa+\kappa^{2} / 2\right)^{1 / 2} \exp (-\kappa),
$$

Let us note that the requirement of crystallization is satisfied at values $\Gamma^{*}>170$ and $\kappa<7$. Apparently from figure 1, calculation under the equation (3) gives a smaller change of critical value $\Gamma_{\mathrm{c}}^{*}$.

\section{Plasma with the condensed disperse phase}

The grains in the combustion plasma are usually of different types and sizes, and thus of different charges. The question remains unanswered: for which subsystem the coupling parameter 
equation (3) is realizable in such a system? As it has been shown in [18], the electrostatic interaction in thermal plasma is missing at the distances exceeding 8 screening lengths. Therefore, if the spatial distribution of smoke grains satisfies this requirement, all grains are electroneutral one to another due to strong screenings. Moreover, we should note that numerical modelling of the crystallization phase diagram [8-11] does not take the grain sizes into consideration. In real systems the structural parameter cannot accept zero value. The Wigner-Seitz radius should be larger than the radius of dust grain $R_{\mathrm{W}}>a$, and the screening length has a terminating value. Hence, there is some minimal value of the structural parameter $\kappa_{a}=a / r_{\mathrm{D}}$, corresponding to the closely-packed structure of the dust grains.

Let us calculate the charge of grains corresponding to the line of fusion of plasma crystal in figure 1, using equation (1). The dependence of the grains charge critical value on structural parameter is presented in figure 2 .

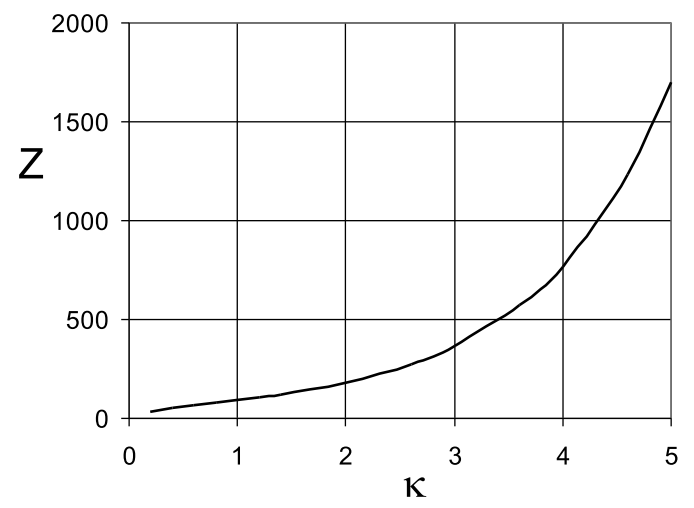

Figure 2. The value of the grains charge, corresponding to line of fusion, at the temperature $T=0.2 \mathrm{eV}$.

Let us take advantage of expression [19]

$$
Z_{\mathrm{d}}=\frac{8 \pi \varepsilon_{0} a^{2} T}{\mathrm{e}^{2} r_{\mathrm{D}}} \sinh \left(\frac{\mathrm{e} \phi_{s}}{2 T}\right)
$$

in order to define the superficial potential of grains corresponding to the charges in figure 2 . We set the grain radius as a part of the Wigner-Seitz radius $a=R_{\mathrm{W}} / m$, which is equivalent to a relation $\kappa_{a}=\kappa / m$.

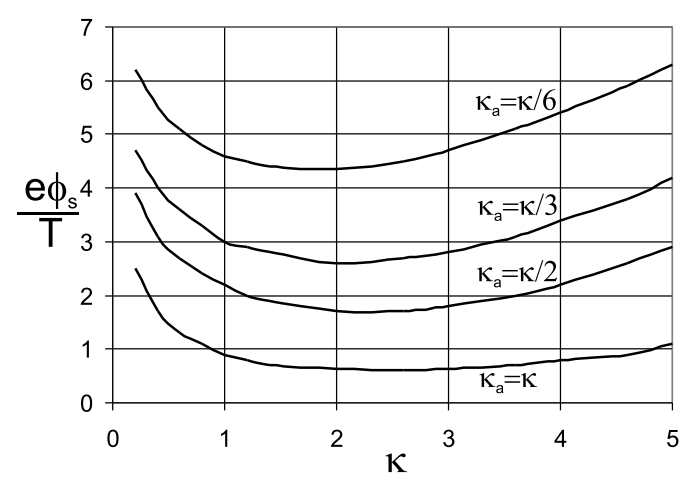

Figure 3. The values of the surface potential of the grains, corresponding to the fusion line at temperature $T=0.2 \mathrm{eV}, r_{\mathrm{D}}=0.3 \mu$ (data from [11,14]).

In figure 3 the dependences of the surface potential of the condensed grains, corresponding to the fusion line, on the structural parameter are presented at various values of $m$. Here we can see 
that all dependences have a minimum in the region of values $\kappa \sim 2-3$. Proceeding from aspiration of the system to a minimum of energy, it is possible to consider such a value of structural parameter as optimum for a plasma crystal.

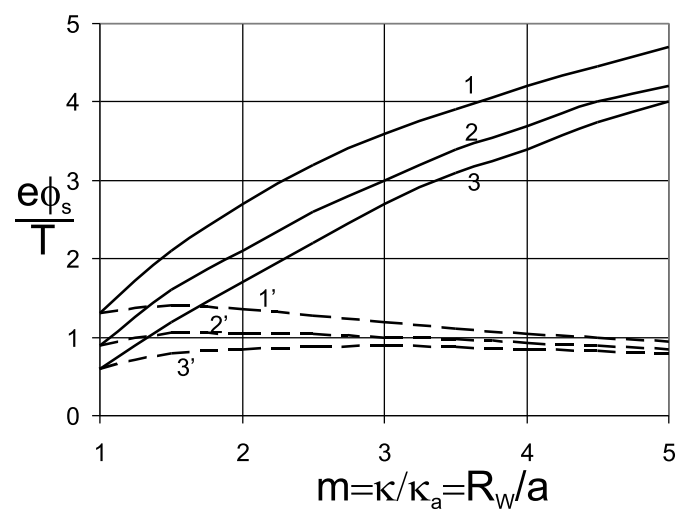

Figure 4. The dependence of the surface potential of grains on the relative Wigner-Seitz radius 1: for $\kappa=0.6$; 2 : for $\kappa=1.3 ; 3$ : for $\kappa=2.6$; and $1^{\prime}, 2^{\prime}, 3^{\prime}$ is the $\mathrm{e} \phi_{s} / m T$.

The dependence of the surface potential on the grains radius (figure 4, curves 1-3) is obvious, because with diminution of the grains radius, the distance between the surfaces of the grains should increase at constant value of $\kappa$. Hence, a larger magnitude of the potential is necessary in order to maintain the force of interaction between grains. Zero value of the surface potential must correspond to the value $\kappa_{a}=\kappa$, since in this case $R_{\mathrm{W}}=a$ and the grains adjoin the surfaces, forming a closely-packed arrangement. The existence of nonzero potential for $\kappa_{a}=\kappa$ testifies to essential restrictions of applicability of the coupling parameter in the form of equations (1)-(3) to the real systems containing grains of terminating size. The surface potential, providing the interaction in the crystal, increases with the increase of $m=\kappa / \kappa_{a}$. However, the ratio e $\phi_{s} / m T=a \mathrm{e} \phi_{s} / R_{\mathrm{W}} T$ (curves $1^{\prime}-2^{\prime}$, figure 4 ) changes a little and aspires to unity with increase of $m$.

It is possible to note, that in equation (1) the charge of particle with radius $a$ defines the Coulomb potential at a distance $R_{\mathrm{W}}$ from the particle surface $\phi\left(R_{\mathrm{W}}\right)=\mathrm{e} Z / 4 \pi \varepsilon_{0} R_{\mathrm{W}}$. Hence, the parameter equation (1) can be written down as follows:

$$
\Gamma=\frac{\mathrm{e} Z_{\mathrm{d}} \phi\left(R_{\mathrm{W}}\right)}{T}
$$

When the screening effect occurs it is also possible to use equation (5). However, it is necessary to calculate the potential proceeding from the solution of the Poisson equation. For example, definition of the surface potential of the large grains $\left(a \gg r_{\mathrm{D}}\right)$ should use equation (4), which can also be dilated for the case of fine grains $\left(a<r_{\mathrm{D}}\right)[20]$ :

$$
\phi_{s}=2 \frac{T}{\mathrm{e}} \operatorname{asinh}\left(\frac{\mathrm{e}^{2} Z_{\mathrm{d}}}{8 \pi \varepsilon_{0} T r_{\mathrm{D}} \kappa_{a}\left(\kappa_{a}+1\right)}\right) .
$$

Following Ikezy it is impossible to use the Debye potential being the potential of the grain surface that corresponds to fusion line. The analysis of the possible solutions of the Poisson equation [21] has shown that at larger values of the surface potential $\left(\phi_{s} \rightarrow \infty\right)$ the curvature of the grain appears incidental, and the potential spatial distribution around the grain can be described by the following expression $[19,20]$

$$
\phi\left(R_{\mathrm{W}}\right)=\frac{2 T}{\mathrm{e}} \ln \left[\frac{1+\tanh \left(\frac{\mathrm{e} \phi_{s}}{4 T}\right) \exp \left(\kappa_{a}-\kappa\right)}{1-\tanh \left(\frac{\mathrm{e} \phi_{s}}{4 T}\right) \exp \left(\kappa_{a}-\kappa\right)}\right] .
$$


The dependences of the coupling parameter, calculated using the equations (5)-(7), on the structural parameter along a fusion line of the plasma crystal for various values of $m=R_{\mathrm{W}} / a=$ $\kappa / \kappa_{a}$ are presented in figure 5 .

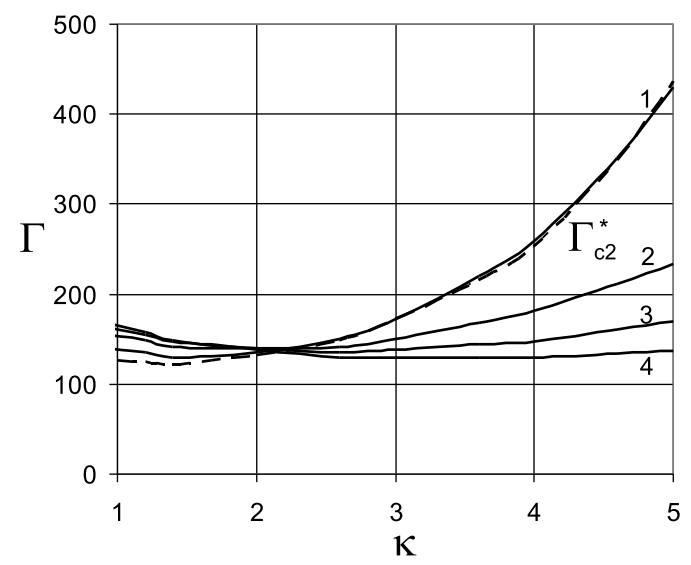

Figure 5. The dependence of the coupling parameter on the structural parameter; 1 : for $m=2$; 2 : for $m=3$; 3 : for $m=4 ; 4$ : for $m=5$.

From the presented dependences it follows that the coupling parameter calculated using the formulas of equations (5)-(7) coincides well with the asymptotics of equation (3) when $R_{\mathrm{W}} \sim 2 a$. The further magnification of coefficient $m$ leads to the impairment of dependence of the coupling parameter on the structural parameter. The relation $R_{\mathrm{W}} \sim 4 a$ (curve 3 ) may be optimum, because in this case the coupling parameter changes a little with the change of the structural parameter.

Thus, the Coulomb coupling parameter, introduced for the one-component plasma, is applicable to the complex plasma if it is used in the form of equation (5). In this case, the value of potential on the boundary of the Wigner-Seitz cell should be taken from the solution of the electrostatic task corresponding to the type and composition of plasmas.

\section{The polydisperse systems}

So far this was a question to be resolved in a monodisperse system of dust grains. When plasma contains the grains of different size or different chemical composition, equation (5) can be applied only to the interaction of the neighbouring grains of two sorts. If there are more than two sorts of grains it is necessary to introduce the coupling parameters for interaction of each sort of grains with all others sorts. For example, if plasma contains the dust grains of three sorts [20,22] it is necessary to use three different coupling parameters, which makes the use of coupling parameter inconvenient as a measure of formation of the ordered structures in the plasma.

Possibly, in such cases there is no need to describe the interaction of the condensed grains among themselves, but concentrate on the interaction of grains with plasma. So, in the smoky plasma which is of thermal collision nature, the interaction of grains is defined by the ion interface pressure in the thin layer of plasma at the grain surface [20,23]. These forces are caused by nonhomogeneous ionization of a gas phase which is described by the modernized Saha equation [20-23]

$$
\frac{n_{\mathrm{e}} n_{\mathrm{i}}}{n_{a}}=K_{\mathrm{S}} \exp \left(\frac{-\mathrm{e} \varphi_{\mathrm{pl}}}{T}\right)
$$

where $K_{\mathrm{S}}$ is the Saha constant, $\varphi_{\mathrm{pl}}$ is the bulk plasma potential.

The bulk plasma potential $\varphi_{\mathrm{pl}}$ characterizes electrostatic energy of the volume of plasma [18]

$$
\varphi_{\mathrm{pl}} Q_{\mathrm{pl}}=\varepsilon_{0} \int_{V} E^{2} \mathrm{~d} V
$$


where $E$ is the electric field, $Q_{\mathrm{pl}}$ is the charge of the plasma volume. Thus the potential $\phi$ is calculated with respect to the bulk plasma potential.

Then the coupling parameter can be defined as the ratio between electrostatic energy in the Wigner-Seitz cell $\varphi_{\mathrm{pl}} Q_{\mathrm{pl}}$ and the thermal energy. The volume of the cell will be calculated with respect to the screening length $r_{\mathrm{D}}$ :

$$
\Gamma_{\mathrm{pd}}=\frac{Q_{\mathrm{pl}} \varphi_{\mathrm{pl}}}{\left(R_{\mathrm{W}} / r_{\mathrm{D}}\right)^{3}} \frac{1}{T} .
$$

In equation (9) the value $Q_{\mathrm{pl}} / R_{\mathrm{W}}^{3}$ is equal to the charge density of a gas phase in the WignerSeitz cell. Hence,

$$
\frac{Q_{\mathrm{pl}}}{R_{\mathrm{W}}^{3}} r_{\mathrm{D}}^{3}=\mathrm{e}\left(n_{\mathrm{i}}-n_{\mathrm{e}}\right) r_{\mathrm{D}}^{3}=\mathrm{e}\left(N_{\mathrm{i}}-N_{\mathrm{e}}\right)_{\mathrm{D}}
$$

represents the volumetric charge of the Debye sphere. Thus, the coupling parameter can be presented in the following form

$$
\Gamma_{\mathrm{pd}}=\left(N_{\mathrm{i}}-N_{\mathrm{e}}\right)_{\mathrm{D}} \frac{e \varphi_{\mathrm{pl}}}{T} .
$$

As it follows from the above expression, the coupling parameter does not depend on the grains number densities, because equation (10) does not contain the Wigner-Seitz radius. On the other hand, equation (10) contains the bulk plasma potential, which is the average value for the whole plasma volume. Therefore, parameter $\Gamma_{\mathrm{pd}}$ can be used as a measure of nonideality of polydisperse system of condensed grains in the plasma with condensed disperse phase. Equation (10) can also be used for the flat electrode, such as a probe, in the plasma.

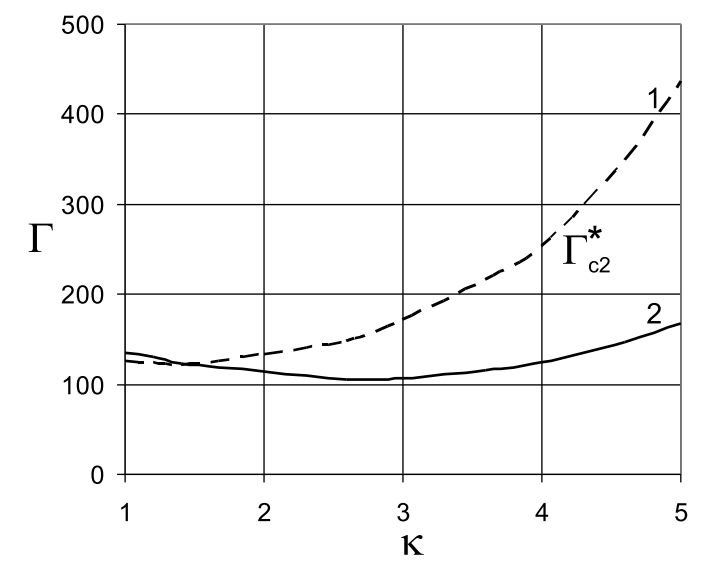

Figure 6. The dependence of the coupling parameter on the structural parameter; 1 - equation (3), 2 - equation (10).

The lines of fusion of the plasma crystal are similar to those presented in figure 5 , but calculated using equation (3) and equation (10) in figure 6 . In the diagrams it is seen that the parameter $\Gamma_{\mathrm{pd}}$ is more preferable for the monodisperse grains as well because its value to a less degree depends on the structural parameter.

\section{Conclusion}

Thus, we can draw a conclusion that the coupling parameter in equation (10) is applicable to the description of polydisperse systems of condensed grains in complex plasma. This form of coupling parameter contains the average electrostatic energy of the system as a product of the average charge of a gas phase into the Debye sphere and the bulk plasma potential. Therefore, equation (10) is independent of the size and charge of the single grains, but depends on the average charge of all the grains. 
In order to describe the system of the monodisperse grains in the complex plasma it is possible to use the coupling parameter in equation (10) or the coupling parameter in equation (5). This form is similar to the coupling parameter for the one-component plasma in equation (1), but the potential on the boundary of the Wigner-Seitz cell should be taken as a solution of the Poisson equation corresponding to the type and composition of the plasma.

In the one-component plasma, the potential on the boundary of the Wigner-Seitz cell is the Coulomb potential, and therefore equation (5) is transformed into equation (1).

\section{References}

1. Chu J.H., Lin I. Phys. Rev. Lett., 1994, 72, No. 25, 4009-4012.

2. Fortov V.E., Khrapak A.G., Khrapak S.A., Molotkov V.I., Petrov O.F. Phys. Usp., 2004, 174, No. 5, 495-544.

3. Dragan G.S., Mal'gota A.A., Protas S.K., Smaglenko T.F., Sokolov Yu.V. - In: Proc. of the scientific and technical meeting of Comecon member countries, Alma-Ata, USSR, 25-31 October, 1982 (Institute of High Temperatures of the USSR Academy of Sciences (IVTAN), Moscow, 1984) p. 191-192 (in Russian).

4. Fortov V.E., Nefedov A.P., Petrov O.F., Samarian A.A., Chernyschev A.V., Lipaev A.M. Pis'ma v ZhETF, 1996, 63, No. 3, 176-180.

5. Pollock E.L., Hansen J.P. Phys. Rev. A., 1973, 8, No. 6, 3110-3122.

6. Ichimaru S. Rev. Mod. Phys., 1982, 54, No. 4, 1017-1059.

7. Ikezi H. Phys. Fluids., 1986, 29, No. 6, 1764-1766.

8. Hamaguchi S., Farouki R.T. J. Chem. Phys., 1994, 101, No. 11, 9876-9884.

9. Farouki R.T., Hamaguchi S. J. Chem. Phys., 1994, 101, No. 11, 9885-9893.

10. Hamaguchi S., Farouki R.T., Dubin D.H.E. J. Chem. Phys., 1996, 105, No. 17, 7641-7647.

11. Hamaguchi S., Farouki R.T., Dubin D.H.E. Phys. Rev. E, 1997, 56, No. 4, 4671-4682.

12. Vishnyakov V.I., Dragan G.S. Ukr. J. Phys., 2004, 49, No. 2, 132-136.

13. Mohideen U., Rahman H. U., Smith M. A., Rosenberg M., Mendis D.A. Phys. Rev. Lett., 1998, 81, No. 2, 349-352.

14. Vaulina O.S., Khrapak S.A. JETP, 2000, 90, No. 2, 287-289.

15. Shukla P.K. Phys. Rev. Lett., 2000, 84, No. 23, 5328-5330.

16. Vaulina O., Khrapak S., Morfill G. Phys. Rev. E, 2002, 66, No. 1, 016404.

17. Vaulina O.S., Vladimirov S.V. Phys. Plasmas, 2002, 9, No. 3, 835-840.

18. Vishnyakov V.I., Dragan G.S. Phys. Rev. E., 2005, 71, No. 1, 016411.

19. Yakubov I.T., Khrapak A.G. Sov. Tech. Rev. B. - Therm. Phys., 1989, 2, 269-337.

20. Vishnyakov V.I., Dragan G.S. Phys. Rev. E., 2006, 73, No. 2, 026403.

21. Vishnyakov V.I., Dragan G.S., Evtuhov V.M., Margaschuk S.V. Teplofiz. Vys. Temp. [High Temp. (USSR)], 1987, 25, No. 3, 620 (in Russian).

22. Vishnyakov V.I., Dragan G.S. Cond. Matter Phys., 2003, 6, No. 4. 687-692.

23. Vishnyakov V.I. Phys. Plasmas, 2005, 12, No. 10, 103502. 


\section{Параметр неідеальності для низькотемпературної плазми з конденсованою фазою}

\section{В.І.Вишняков, Г.С.Драган}

Фізичний факультет Одеського національного університету ім. І.І. Мечникова, 65026, Одеса, Україна

Отримано 6 березня 2006 р., в остаточному вигляді - 18 травня 2006 р.

Досліджено межі застосування параметра неідеальності та його вираз для опису фазових переходів в упорядкованих структурах заряджених конденсованих часток в плазмі з конденсованою дисперсною фазою. Показана доцільність використання виразу для параметру неідеальності із застосуванням значення потенціалу на границі комірки Вігнера-Зейтца, отриманого з розв'язку електростатичної задачі, що відповідає виду і складу плазми. Отримано вираз параметра неідеальності для полідисперсної конденсованої фази з електростатичною енергією міжфазної взаємодії у вигляді добутку узагальненого потенціалу плазми і об'ємного заряду газової фази плазми в дебаєвській сфері.

Ключові слова: плазма, конденсована дисперсна фаза, параметр неідеальності

PACS: 52.27.Gr, 52.27.Lw 